\title{
Radar Beam Occultation Studies Using GIS and DEM Technology: An Example Study of Guam
}

\author{
PAul A. Kucera \\ Department of Atmospheric Sciences, University of North Dakota, Grand Forks, North Dakota \\ WiTOLD F. KRAJEWSKI \\ IIHR-Hydroscience \& Engineering, The University of Iowa, Iowa City, Iowa \\ C. BRYAN YOUNG \\ Department of Civil, Environmental, and Architectural Engineering, University of Kansas, Lawrence, Kansas
}

(Manuscript received 23 June 2003, in final form 9 February 2004)

\section{ABSTRACT}

\begin{abstract}
Geographic information systems (GISs) combined with digital elevation models (DEMs) provide opportunities to evaluate weather radar beam blockage and other ground clutter phenomena. The authors explore this potential using topographic information and a simple beam propagation model for the complex terrain of Guam. To evaluate the effect of different DEM resolutions, they compare the simulated patterns of complete and partial beam blockage with probability of detection maps derived from a large database of level II radar reflectivity for the U.S. Air Force Weather Surveillance Radar-1988 Doppler (WSR-88D) on Guam. The main conclusion of the study is that the GIS approach provides useful insight into the actual pattern of blocked areas. The DEM resolution plays a role in resolving the blocked patterns. In general, higher DEM resolution provides better results although widely available lower-resolution DEMs can provide valuable information about beam-blocking effects.
\end{abstract}

\section{Introduction}

Understanding the extent to which a radar view is blocked by surrounding obstacles plays an important role in the proper interpretation of reflectivity data. For example, users of radar data collected for hydrological and meteorological applications need to know the limitations of rainfall estimates or forecasts associated with blockage (e.g., Pellarin et al. 2002). Such information is not readily available, even though radar horizon maps are determined for a particular location while designing a radar network. In addition, anticipated beam blockage often affects the development of radar scanning strategies for a particular site.

It is well known that in many mountainous regions of the United States, the national network of operational Weather Surveillance Radar-1988 Doppler (WSR-88D) radars suffers from significant blockage (e.g., Westrick et al. 1999; Nelson et al. 2003; Young et al. 1999). However, a casual user of radar data and products does

Corresponding author address: Paul A. Kucera, Dept. of Atmospheric Sciences, University of North Dakota, P.O. Box 9006, Grand Forks, ND 58202.

E-mail: pkucera@aero.und.edu not know the exact locations and severity of the problem for a particular case of interest. Our objective is to demonstrate that a geographic information systems (GISs) based analysis can reveal terrain effects for radar data applications. In this paper we present a methodology that many nonradar experts can use to determine potential problems associated with beam blockage. We have developed GIS-based software for radar beam propagation and tested it using an extensive database for Guam. We have collected a large dataset of radar reflectivity (level II) data and a high-resolution (10 m in the horizontal) digital elevation data map that facilitates this analysis.

Guam is a $550 \mathrm{~km}^{2}$ island centered at approximately $13^{\circ} 25^{\prime} \mathrm{N}, 144^{\circ} 45^{\prime} \mathrm{E}$ in the western North Pacific (see Fig. 1). It is a 50-km-long, elongated-shaped, northeastsouthwest-oriented island about $18 \mathrm{~km}$ wide at the northern and southern ends and $6.4 \mathrm{~km}$ wide at the middle. The northern half of the island is a relatively flat, uplifted limestone plateau ranging in elevation from 80 to $220 \mathrm{~m}$. There are two significant hills on the plateau, Mount Santa Rosa and Mount Barrigada. The southern half of the island is composed of basaltic mountains and hills, with a maximum elevation of 


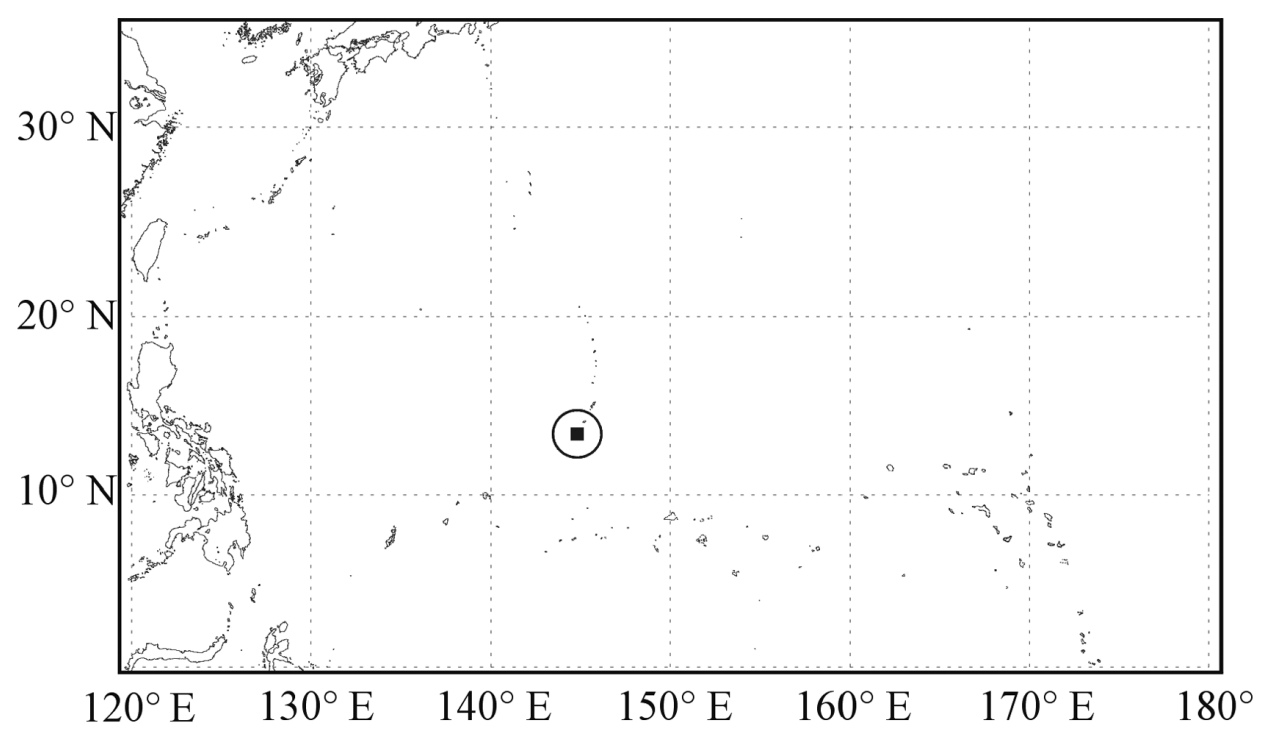

FIG. 1. Map of the northwestern Pacific Ocean basin. The outer circle surrounding Guam shows the maximum coverage of the Guam radar.

$406 \mathrm{~m}$ and with five peaks exceeding $330 \mathrm{~m}(1000 \mathrm{ft})$. For the interested reader, a detailed description of the geological characteristics of Guam and surrounding islands is given by Ward et al. (1965).

The complex terrain of Guam has caused problems in rainfall data collection for the National Aeronautics and Space Administration (NASA) Tropical Rainfall Measuring Mission (TRMM; Simpson et al. 1988, 1996; Kummerow et al. 2000). A WSR-88D radar was deployed on Guam for the needs of the Andersen Air Force Base and not rainfall monitoring. Its view toward Rota Island, where TRMM has a dense rain gauge cluster (Krajewski et al. 2003), is blocked and so is a wide sector toward the south and west. Kucera (2002) studied the impact of this blocking on radar rainfall estimation and monitoring.

The paper is composed of six main sections. In section 2 we describe the data in detail, and follow this with a discussion of the beam propagation simulation methodology in section 3 . We describe our methodology of evaluating the simulation using actual data analysis in section 5 . We close the paper with a set of conclusions in section 6.

\section{Data}

\section{a. Weather Surveillance Radar reflectivity}

Weather radar data collected on Guam were recorded by an operational WSR-88D radar, which is part of the U.S. Next-Generation Weather Radar (NEXRAD) network (Heiss et al. 1990; Crum et al. 1993, 1998). WSR$88 \mathrm{D}$ radars transmit at a wavelength around $10 \mathrm{~cm}$ (frequency of $2.9 \mathrm{GHz}$ ) and transmit horizontally polarized electromagnetic radiation at a peak power of $475 \mathrm{~kW}$. WSR-88D radars have a high-resolution half-power
(3 dB) beamwidth of $0.95^{\circ}$. The pulse repetition frequency of the radar ranges from 320 to $1000 \mathrm{~Hz}$ for two fixed pulse widths of 1.57 and $4.5 \mu \mathrm{s}$. Data are collected and stored in spherical coordinates (range, azimuth, elevation). The radar has a nominal range resolution of $1 \mathrm{~km}$ and an azimuth resolution of $1^{\circ}$.

The WSR-88D radar on Guam (NEXRAD identifier PGUA) is located on the east-central side of the island (see Fig. 2). It was commissioned on 22 October 1992. The WSR-88D radar is operated and maintained by Andersen Air Force Base (AAFB) personnel. It collects three moments of the returned signal: radar reflectivity, mean Doppler velocity, and Doppler velocity spectral width. The Guam WSR-88D data are recorded and stored in a raw product format called level II archive data. These products are digitally stored to 8-mm magnetic tapes and sent to the National Climatic Data Center (NCDC). We have obtained the level II data by two avenues. First, we obtained all existing Guam data at NCDC through an agreement with the NASA TRMM office. After the TRMM satellite was launched, AAFB weather office personnel, in collaboration with the University of Guam, made copies of the archive tapes at the site and sent them to the University of Iowa. To provide fast, efficient access to the entire dataset, we store radar reflectivity data online in an ASCII run-length encoded (RLE) format that was designed for radar data and described by Kruger and Krajewski (1997).

Our study encompasses Guam WSR-88D radar data collected between December 1995 and August 2000. Guam has numerous problems that require various quality control and masking algorithms to exclude compromised WSR-88D data from ground validation analysis. We processed all the radar volume scans between 1995 


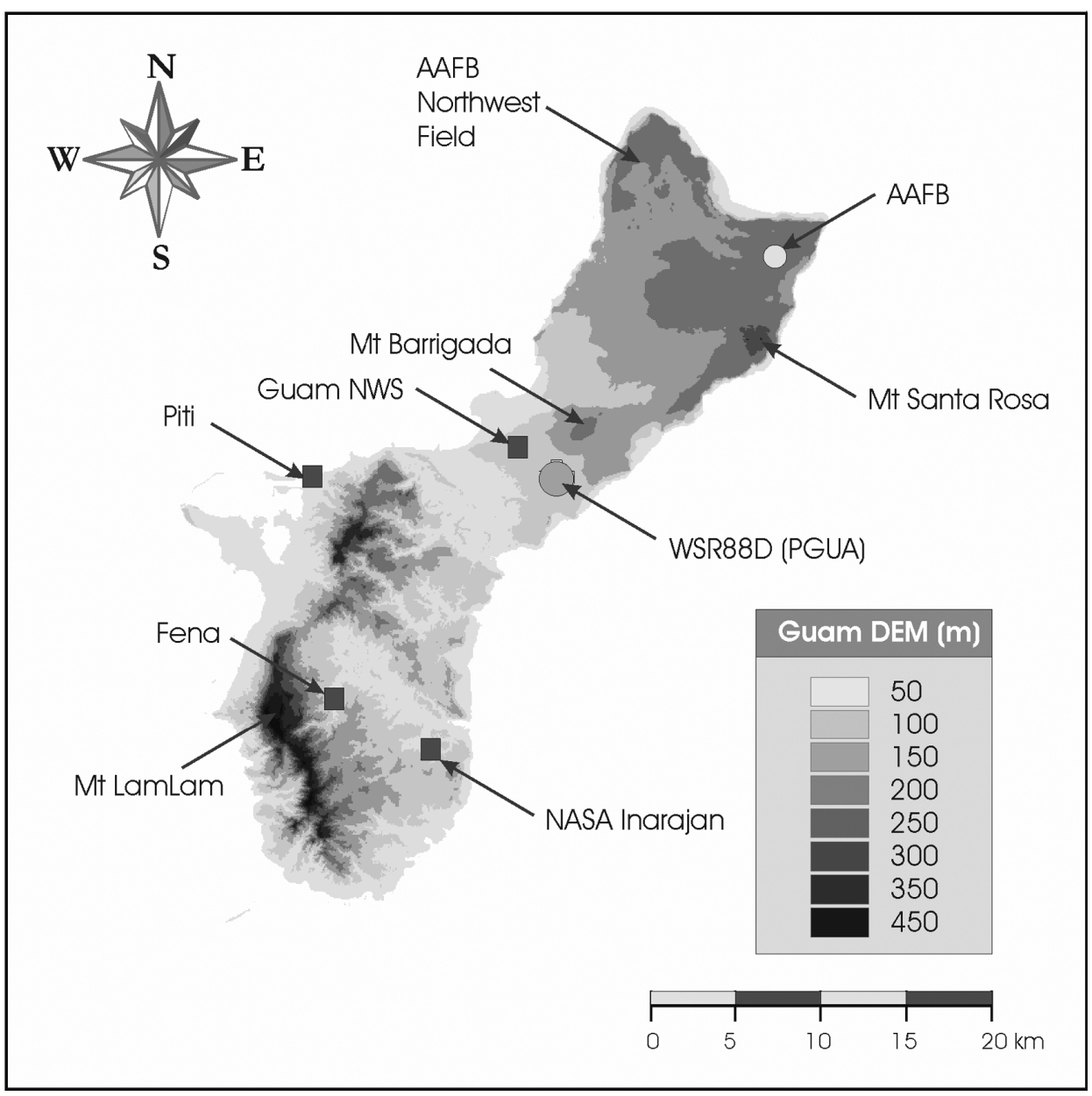

FIG. 2. Elevation map of Guam showing the location of the WSR-88D and rain gauges.

and 2000 to examine the long-term spatial patterns of observed reflectivity to see what sectors might be usable for analysis.

To assess the patterns of the partial and complete radar beam occultation we calculated fields of the probability of detection (POD). In this work we define the POD as the ratio of the number of radar observations exceeding a certain threshold of reflectivity to the total number of observations (i.e., scans) taken at a given location. Using the POD over a long period, regions of sea clutter, ground clutter, and beam blockage are easily detectable and identified. To understand the significance of the POD maps, we compared them with results of a radar beam propagation model (cf. section 5 ). The model incorporates a United States Geological Survey (USGS) digital elevation model (DEM) to compare the correspondence of the ground clutter and beam-blocked sectors with the location of terrain features. Using the POD and model results, a product map can be created to exclude regions where the full signal from a meteorological target cannot be obtained.

\section{b. Atmospheric soundings}

Atmospheric sounding data were also analyzed to examine the characteristics of the atmosphere during different climatological conditions and examine the sensitivity of using the standard refraction for radar beam propagation. Sounding data for the years 1997-2000 were obtained from the database maintained by the Atmospheric Science Department at the University of Wyoming. Nominally, soundings are launched every $12 \mathrm{~h}$ (synoptic period) at 0000 and 1200 UTC.

\section{Radar beam propagation model results}

To assess the severity of the blockage and to create a product map for TRMM rainfall validation comparisons, we developed a radar beam propagation model that incorporates DEM data. The model calculates the relative power loss as a function of range, elevation, and azimuth of the radar beam as it interacts with the terrain features stored in the DEM data. The USGS has published a DEM manual (USGS 2000) that gives de- 
tailed descriptions of the different model resolutions, DEM data collection methods, data characteristics for various regions around the world, format of the data records, and the accuracy of the DEM data. The horizontal resolution of the Guam DEM data is $10 \mathrm{~m}$, which is relatively high in comparison to most DEM datasets (30- and 100-m resolution). Data accuracy is reported to be one-half a contour interval or better, which corresponds to $<5 \mathrm{~m}$. We also investigated the effect of using the 30 - and 100-m resolution DEM data by resampling the $10-\mathrm{m}$ resolution data.

The beam propagation model assumes a standard refractive atmosphere (Battan 1973) to calculate the height of the beam as a function of range. The height of the beam can be estimated by the following equation (Rinehart 1991):

$$
h=\sqrt{r^{2}+R^{\prime 2}+2 r R^{\prime} \sin \left(\theta_{e}\right)}-R^{\prime}+h_{0},
$$

where $h$ is the height above a reference point (e.g., ground level, mean sea level, etc.), $r$ is the slant range from the radar, $R^{\prime}$ is the effective earth's radius to account for atmospheric refraction, $\theta_{e}$ is the elevation angle of the radar beam, and $h_{0}$ is the height of the antenna above the reference point. The effective radius, $R^{\prime}$, can be estimated by assuming that the change in refractive index with height in the lower atmosphere is constant. With this assumption, $R^{\prime}$ is approximately $8500 \mathrm{~km}$. The standard refractive atmosphere assumption will be evaluated in later sections.

The model also assumes that surface features (trees, buildings, etc.) that are not represented in the DEM model do not contribute significantly to the scattering of the radar beam power. If the surface features in the vicinity of the radar were known accurately, characteristic roughness lengths could be determined and incorporated into the model.

We assumed that when a portion of the beam was lower than the terrain, the power in the portion of the beam below the surface height was scattered back (ground clutter), absorbed by the surface, or scattered in a direction perpendicular to the path of the beam and no power was scattered in the forward direction. In reality, some of the power would also be scattered forward and contribute to the received power beyond the beam blockage. Because of the complexity of the interaction of electromagnetic waves with terrain, this amount of forward-scattered power is unknown and therefore was not considered in our model.

To evaluate the integral of power within a beam, a common approximation is to assume that the distribution of power within the main lobe can be represented by a two-dimensional Gaussian illumination function (Probert-Jones 1962; Donaldson 1964; Meneghini and Kozu 1990), which is given by the following equation:

$$
f(\theta, \phi)=\exp \left\{-\ln 2\left[\left(2 \frac{\theta}{\theta_{3}}\right)^{2}+\left(2 \frac{\phi}{\phi_{3}}\right)^{2}\right]\right\}
$$

where $\theta_{3}$ and $\phi_{3}$ are the half-power or 3-dB beamwidths along the principal axes [defined to be the angular distance across the main lobe where the power is reduced by one-half of the peak power at the point of maximum gain, $f(\theta, \phi)=0.5]$. The azimuth angles $\theta$ and $\phi$ are measured from the point of maximum gain. The azimuth angles are multiplied by two to account for the angles being only half the total angular distance across the beam. If the antenna is symmetric (i.e., forms a circular beam), which is the case for WSR-88Ds, the Gaussian illumination function can be simplified to

$$
f(\theta)=\exp \left[-\ln 2\left(2 \frac{\theta}{\theta_{3}}\right)^{2}\right],
$$

where $\theta$ is the polar angle measured from the point of maximum gain.

In the model we neglected sidelobes; that is, we ignore power that is not contained in the main beam. The error in ignoring sidelobes can become significant in special circumstances when strong reflectivity gradients are present within the beam volume or if the antenna reflector is not properly constructed. For example, the region near the melting layer often has a strong gradient of reflectivity, which can cause significant sidelobe effects. On the other hand, this is normally not a problem except at far ranges for rainfall estimation algorithms because the lowest tilts are commonly used, which typically remain below the melting level. In general, a Gaussian beam pattern approximation is a good assumption when the measured power in the sidelobes is significantly smaller than the main lobe. The first sidelobe for WSR-88Ds is reported to be $-27 \mathrm{~dB}$ down (Doviak and Zrnić 1993), which is about 500 times smaller than the main lobe power.

Probert-Jones (1962) compared the Gaussian beam approximation to a well-known class of antenna beam patterns that use complicated Bessel functions to represent both the main lobe and sidelobes. He found the Gaussian approximation for more complicated beam patterns varies by less than $0.64 \mathrm{~dB}$ in comparison in most cases. Other investigators have also shown the Gaussian beam pattern is a good approximation for most meteorological applications (Donaldson 1964; Bogush 1989; Andrieu and Creutin 1995).

We extended the Gaussian beam pattern to a $6-\mathrm{dB}$ beamwidth in our model, which is twice the $3-\mathrm{dB}$ beamwidth of the WSR-88Ds. This captures $93.75 \%$ of the total sensitivity of the radar beam pattern. We divided the beam pattern in the plane perpendicular to the propagation path into small $0.1^{\circ} \times 0.1^{\circ}$ elements to capture the finescale interactions with the DEM data. We used a total of $3 \times 10^{5}$ elements in the integration of beam power. The power in each element is weighted by a Gaussian illumination function such that when all the elements are integrated over the entire beam pattern, the following relationship is satisfied: 


$$
p_{t}=\int_{0}^{\theta_{6}} \int_{0}^{2 \pi} f^{2}(\theta) d^{2} p_{s}(\alpha, \theta) \theta d \theta d \alpha=1,
$$

where $p_{t}$ is the total normalized power. The function $f^{2}(\theta)$ is the two-dimensional normalized form of Eq. (3); $d^{2} p_{s}(\alpha, \theta)$ is the power in each elemental unit, $s$; $\alpha$ and $\theta$ are the angles that define the location of $s$ on the disk being a cross section of the beam; and $\theta_{6}$ represents the integration over the $6-\mathrm{dB}$ beamwidth.

To examine the beam blockage as a function of azimuth and range from the radar, we resampled the DEM data into $0.1^{\circ}$ azimuth and $0.1-\mathrm{km}$ range bins. The bins extended to a maximum range of $30 \mathrm{~km}$ from the Guam radar. In other words, for every $0.1^{\circ}$ in azimuth, there were 300 range bins for a total of $1.08 \times 10^{6}$ resampled DEM data bins over the $360^{\circ}$ azimuth angles.

For every $0.1^{\circ}$ in azimuth, we calculated the relative power remaining in the beam. At every range bin, we integrated the power over the $6-\mathrm{dB}$ beam pattern. If any of the power elements in the beam intersected or fell below the land surface, the power in those elements would be subtracted from the total power available. Once blocked, the power in those elements would no longer contribute to the total power down range. We then integrated the power loss as a function of range to obtain a total power for a particular azimuth angle. Mathematically, following Eq. (4), the power loss at each range can be written as

$$
p_{i}=\int_{0}^{\theta_{u}} \int_{0}^{2 \pi} f^{2}(\theta) d^{2} p_{s}(\alpha, \theta) \theta d \theta d \alpha \leq 1,
$$

where $p_{i}$ is the power loss at the $i$ th range bin and $\theta_{u}$ is the polar angle subtended from the center of the beam to the point where beam remains unblocked for a given angle, $\alpha$, in the beam pattern. Obviously, this would be the $6-\mathrm{dB}$ half-beamwidth $\left(\theta_{u}=\theta_{6}\right)$ if there were no beam blockage. At each range bin, the relative power loss can be defined using logarithmic units:

$$
p_{\operatorname{loss}_{i}}=-10 \log _{10}\left(\frac{p_{i}}{p_{t}}\right) .
$$

Because $p_{i} \leq p_{t}$, the relative power loss, expressed in decibels, is always nonnegative. This is convenient for discussion and graphical presentation of the results. The total power loss along an azimuth equals the power loss at the maximum range of the DEM data. In our study, the maximum range is $30 \mathrm{~km}$ and the DEM bins are spaced every $0.1 \mathrm{~km}$. This is the maximum distance from the Guam radar location where there is terrain above the ocean. Therefore, the total power loss is $p_{\text {loss }_{\text {total }}}=p_{\text {loss }_{300}}$.

We implemented several steps to determine which elements in the beam pattern the terrain blocked. The first step was to determine the relative height difference of the center of the beam to the ground. The height of the antenna is $30 \mathrm{~m}$ above ground level (AGL) and the base of the antenna tower is located $80.5 \mathrm{~m}$ above mean sea level (MSL). At each range bin, the height of the center of the beam is estimated using Eq. (1). The next step calculated the height of each elemental bin in the beam pattern using the center beam height as a reference. The height above MSL is then easily calculated for each beam pattern bin. We calculated the location in relation to the ground for each element in the beam pattern knowing the range and azimuth of the center of the beam.

We then compared the height of each beam element to the DEM data at that location. To provide a better comparison in heights, we fit a cubic spline interpolation to the DEM data in the azimuth directions at each range using the algorithm developed by Press et al. (1992). This provided good estimates of the land height in the regions between the discrete DEM data points. If the beam pattern element was located at the same height or below the elevation of the land, it was flagged as blocked. After all (if any) blocked beam elements are located; we calculated the total power using all the nonblocked beam elements. As we stated earlier, once a beam element is considered blocked, it remains flagged as blocked for the remainder of the propagation path. The beam is examined for additional blockage at the next range step and the power loss is recalculated. We repeated this procedure along the range to obtain the total power loss along that azimuth angle. We applied this algorithm to all the azimuth angles to obtain a twodimensional power loss map and total power loss estimate as a function of the azimuth angle from the radar. We repeated these steps for each elevation sweep until no blockage was detected.

As is clear from the above description, we did not consider partial beam blockage by the beam elements. This could be accomplished following the procedure of Hannesen and Löffler-Mang (1998). We also neglected the effects of beam refraction and multiple reflections, which may happen in mountainous terrain. We revisit these issues in the conclusions section of this paper.

\section{POD maps}

We calculated the POD for all of the sweeps recorded in a radar volume. Figure 3 shows the radar reflectivity POD out to a maximum range of $150 \mathrm{~km}$ for the lowest two sweeps. We used data from all of the collected radar volumes in the analysis for the period May 1995-August 2000. There are about 125000 volume scans available during this period. The POD units are in percentage observations that occurred above a reflectivity threshold of $10 \mathrm{dBZ}$. We chose a $10-\mathrm{dBZ}$ threshold because it represents the nominal reflectivity that would be considered for detectable surface rainfall $\left(\sim 0.1 \mathrm{~mm} \mathrm{~h}^{-1}\right)$ and it reduces contamination from sea clutter.

There are several features in the lowest-elevation POD field worth noting. Even though the threshold reduces sea clutter contamination, its signature is still ob- 

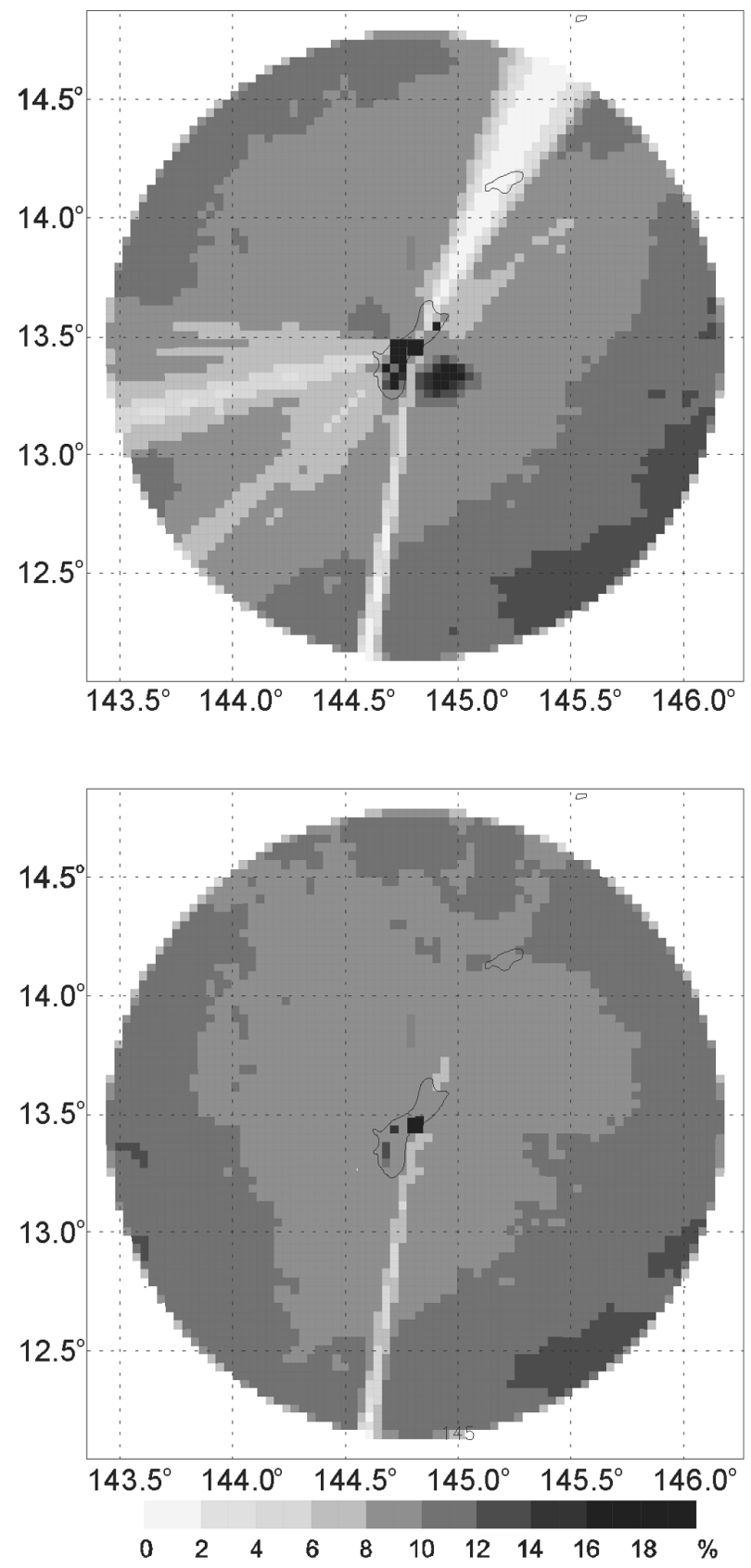

FIG. 3. POD maps for the lowest two sweeps [(top) $+0.5^{\circ}$ and (bottom) $+1.5^{\circ}$ elevation] for radar reflectivity using a threshold of $10 \mathrm{dBZ}$.

served in the POD map. It is the feature that has a relatively high POD $(>18 \%)$ within the first $30 \mathrm{~km}$ east and to a lesser extent to the NNW of Guam. A second feature that is prominent is the high POD associated with the mountainous regions (ground clutter) throughout the island. The locations of the high POD on the island correspond to the mountains on Guam. Behind the mountainous regions is the relatively small POD due to the beam blockage. Severe blockage (close to 100\%) is seen for a sector between azimuths $16^{\circ}$ and $30^{\circ}$, which corresponds to the direction of Rota. A lesser amount of beam blockage is detected at an azimuth of $68^{\circ}$. There is also significant blockage from the mountains to the southwest. The blocked sector encompasses a region bounded by azimuths $212^{\circ}$ and $281^{\circ}$. The last main feature is the entire blockage in a $5^{\circ}$ sector to the south $\left(187^{\circ}-192^{\circ}\right)$. The sector is blocked because of an apartment complex (Ladera Towers), which was built after the radar was installed and is located within $1 \mathrm{~km}$ of the radar. It is interesting to note that the POD in unblocked sectors without significant clutter has a mean POD of about $8 \%$. If one assumes that all of the return detected is from meteorological echo (i.e., rainfall), the Guam region observes rainfall about $8 \%$ of the time.

\section{Model and POD data comparison}

The total model-predicted power loss for the lowest two sweeps is shown in Fig. 4. In the first sweep (antenna elevation angle of $+0.5^{\circ}$ ), over $50 \%$ of the sectors have at least partial blockage. The beam blockage corresponds to locations with lower POD due to beam blockage shown in Fig. 3. Two main blockages are detected with the model. The first blockage is between azimuths $355^{\circ}$ and $75^{\circ}$. The second blocked sector is to the southwest between azimuths $200^{\circ}$ and $285^{\circ}$. The analysis of the second sweep (elevation angle of $+1.5^{\circ}$ ) shows very little beam blockage except for partial beam blockage $(\sim 0.7 \mathrm{~dB})$ in the direction of Rota. In Fig. 5, we show the relative power loss between the $10-\mathrm{m}$ and lower DEM resolutions. The differences in power are apparent especially in the regions with major beam blockages.

It is important to see if the model-predicted beam blockage agrees with radar observations. For this analysis, we plotted the POD and two-dimensional power loss maps on the same Cartesian coordinate system. The resolution of the grid is $4 \mathrm{~km}$ to approximate the Hydrologic Rainfall Analysis Project (HRAP) grid size (Fulton et al. 1998). The two-dimensional DEM blockage map is shown for the lowest-elevation sweep in Fig. 6 (recall the POD map shown in the top panel of Fig. 3 ). The second elevation sweep is not shown because there is very little blockage except for a small amount in the direction of Rota.

The spatial pattern of beam blockage and POD agree quite well. The significant blockages are located along the same azimuths. Some of the less significant beam blockage that is indicated in the DEM model is not prominently seen in the POD. For example, the weak blockage in azimuths $355^{\circ}-15^{\circ}$ (see Fig. 5) is not detected in the POD map (Fig. 4). Also, the weakly blocked sector to the southwest $\left(200^{\circ}-210^{\circ}\right)$ is not a feature detected in the POD analysis.

The last comparison that we performed was to see if a relationship could be formed between power loss estimated by our beam propagation model and the long- 


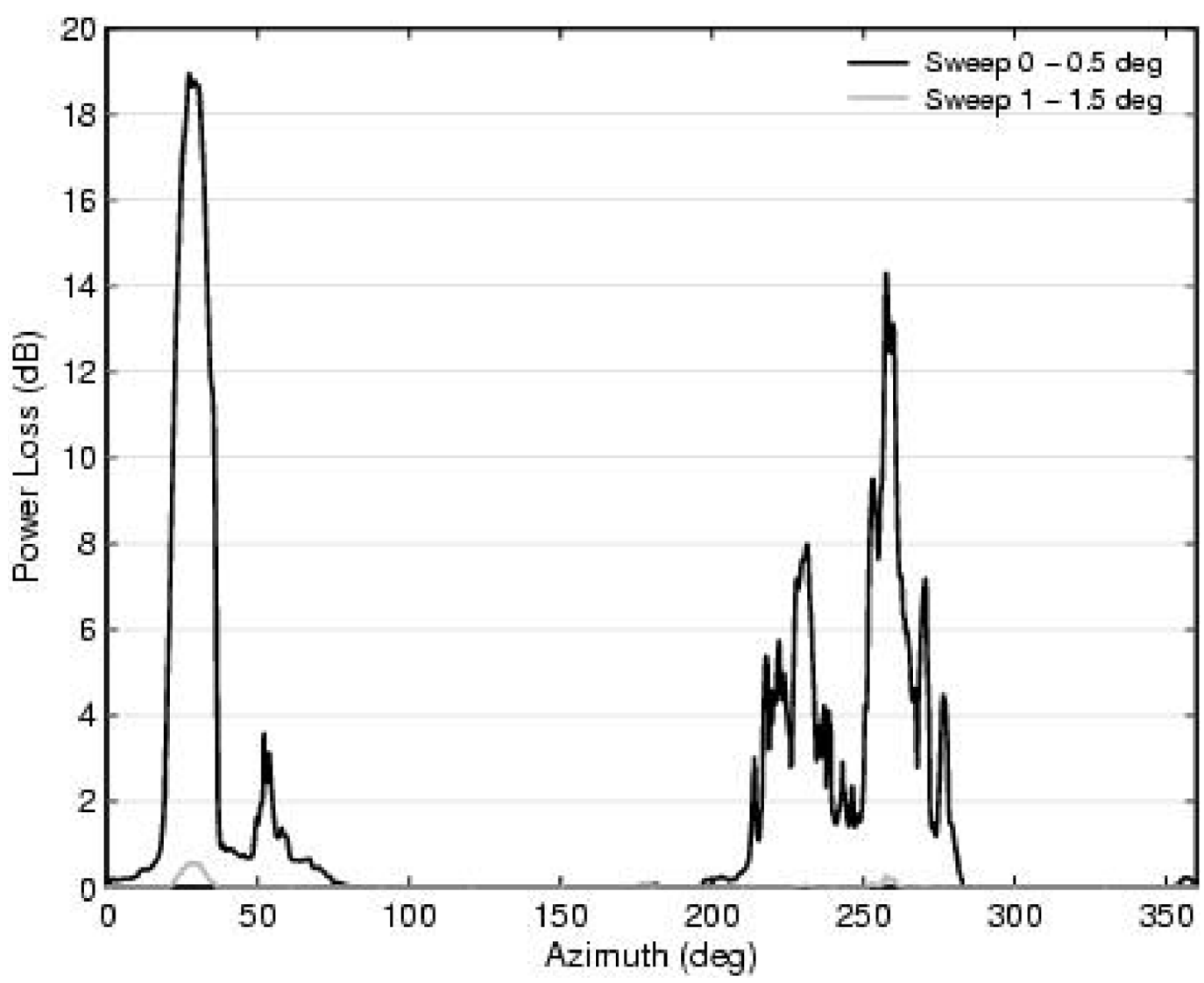

FIG. 4. Estimated power loss due to beam blockage calculated from a beam propagation model and DEM data for the lowest two sweeps $\left(+0.5^{\circ}\right.$ and $+1.5^{\circ}$ elevation $)$. The plot indicates most of the blockage occurs in the first $\left(+0.5^{\circ}\right)$ sweep.

term POD using radar reflectivity data. For this analysis, we compared the total power loss to the POD at $1^{\circ}$ azimuth angles at a fixed range from the radar. From the two-dimensional maps, a range of $50 \mathrm{~km}$ met the criteria for a representative sample. First, this range is just beyond the maximum distance of the island, beyond which the power loss does not increase. The respective POD is outside the range of sea and ground clutter but close enough that range effects are not an issue. The scatterplot of the comparison for 10-, 30-, and 100-m DEM resolution is shown in Fig. 7. Clearly, there is a strong relationship between model-predicted power loss and the decrease in the POD. The points located along the ordinate axis indicate the regions of no beam blockage. The range of values represents the natural variability in POD observed near Guam.

We attempted to describe the relationship between model-predicted power loss and observed POD with a regression model. We compared several regression models and determined that a quadratic model gave the best fit. Because all three resolutions gave very similar model parameters, we only show the regression curve for the 10-m DEM resolution data. The model has the following form:

$$
\mathrm{POD}=-0.0059 P_{\mathrm{loss}}^{2}-0.3086 P_{\text {loss }}+7.642,
$$

where $P_{\text {loss }}$ is the power loss derived from the DEM data. All of the points with no power loss were excluded from the regression analysis. The adjusted $R^{2}$ value for the model was 0.9493 with an rmse of 0.4776 .

Analysis of the lower-resolution DEM data shows that the best agreement between the POD maps and the beam propagation model is for the highest-resolution data. Decreasing the resolution increases the scatter between the two quantities. The corresponding $R^{2}$ values are 0.9438 and 0.9250 , respectively for the 30 - and $100-\mathrm{m}$ resolutions. Based on our study, long-term POD can be a good predictor of regions with power loss or blocked sectors. This information can be used to flag sectors that should be excluded from rainfall analysis.

\section{Beam height due to refraction}

At microwave frequencies, a good assumption is that wave fronts are perpendicular and propagate along the ray path. It can be shown that for a spherically stratified atmosphere that distance along the earth's surface, $s(h)$, 


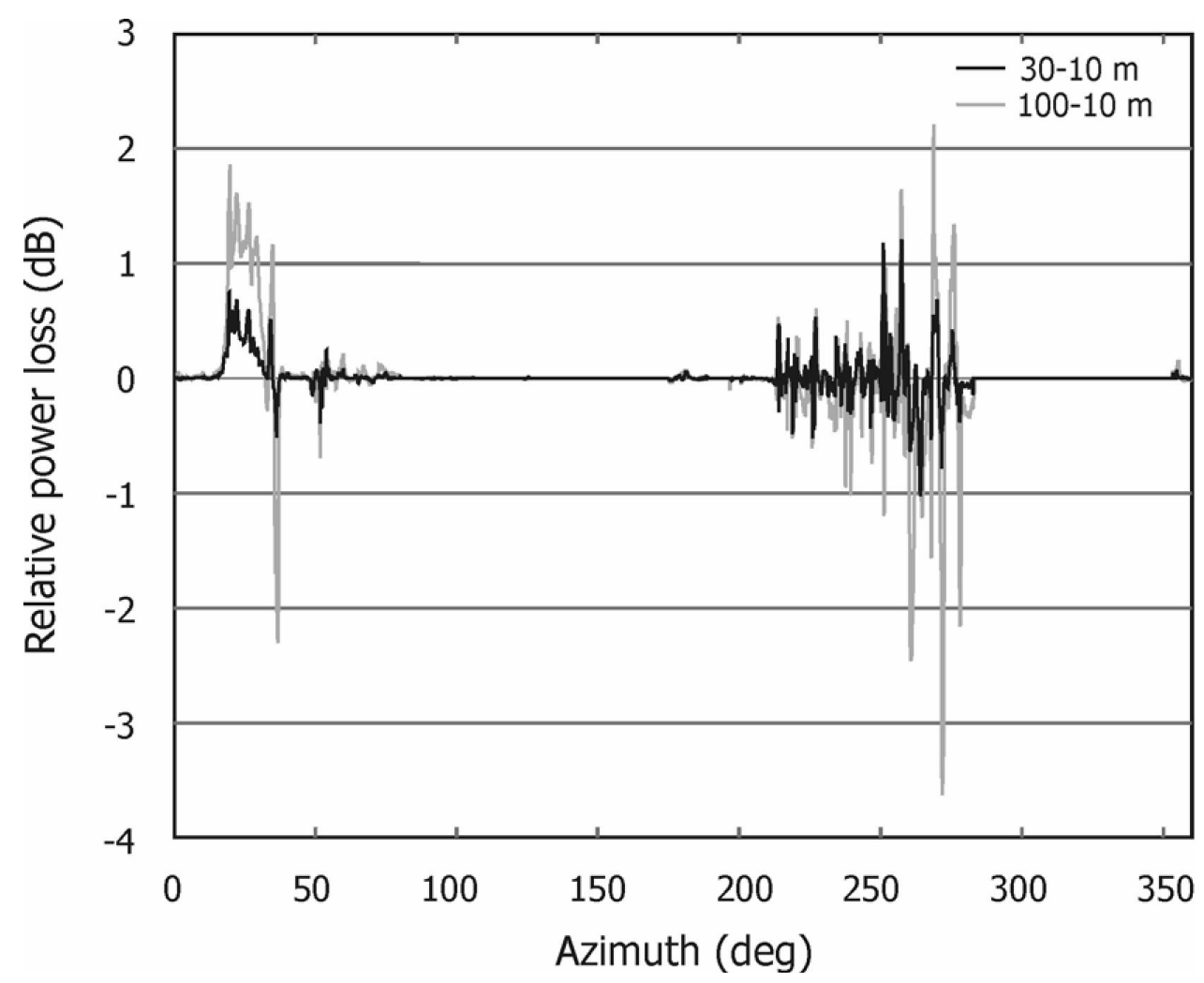

FIG. 5. Relative power loss of 30- (black) and 100-m- (gray) resolution data compared to the power loss using $10-\mathrm{m}$ DEM resolution for the lowest sweep $\left(+0.5^{\circ}\right.$ elevation angle). Positive values indicate lowerresolution DEM data had a lower power loss compared to the 10-m DEM estimates.

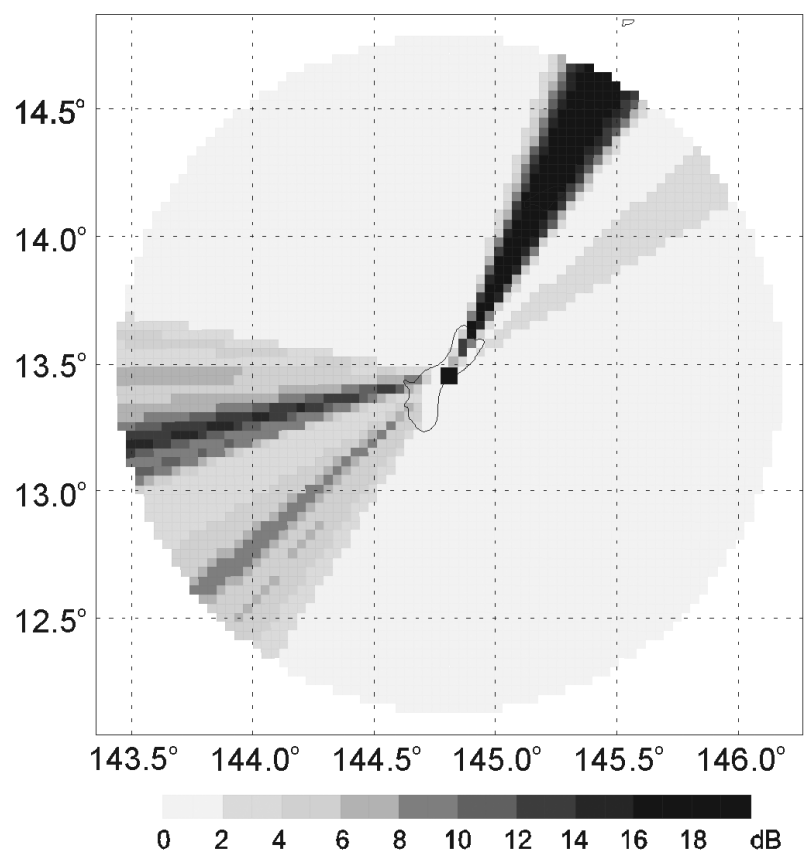

FIG. 6. Two-dimensional power loss map for the first elevation sweep $\left(+0.5^{\circ}\right)$ derived from the beam propagation model using DEM data. below a ray at height $h$ is given by the following equation (Doviak and Zrnić 1993):

$$
s(h)=\int_{0}^{h} \frac{R C d h}{r\left[r^{2} n^{2}(h)-C^{2}\right]^{1 / 2}},
$$

with

$$
C=R n(0) \cos \left(\theta_{e}\right)
$$

where $R$ is the earth's radius, $r$ is the distance from the center of the earth to the location of the ray $(R+h)$, $\theta_{e}$ is the elevation angle above the horizon, and $n(0)$ is the refractive index at the surface (at the radar). The main assumption is that the refractive index, $n(h)$, is smoothly changing for a given wavelength such that ray theory applies. It can be shown that Eq. (8) is a solution to the second-order differential equation (Hartree et al. 1946):

$$
\frac{d^{2} h}{d s^{2}}-\left(\frac{2}{r}+\frac{1}{n} \frac{d n}{d h}\right)\left(\frac{d h}{d s}\right)^{2}-\left(\frac{r}{R}\right)^{2}\left(\frac{1}{r}+\frac{1}{n} \frac{d n}{d h}\right)=0 .
$$

Equation (8) can be solved to obtain an earth-equivalent model by assuming a standard refractive atmosphere (Doviak and Zrnić 1993). This assumption leads to the $4 / 3$ earth approximation. There are times when this assumption does not hold (i.e., temperature inversions, frontal boundaries, etc.). 


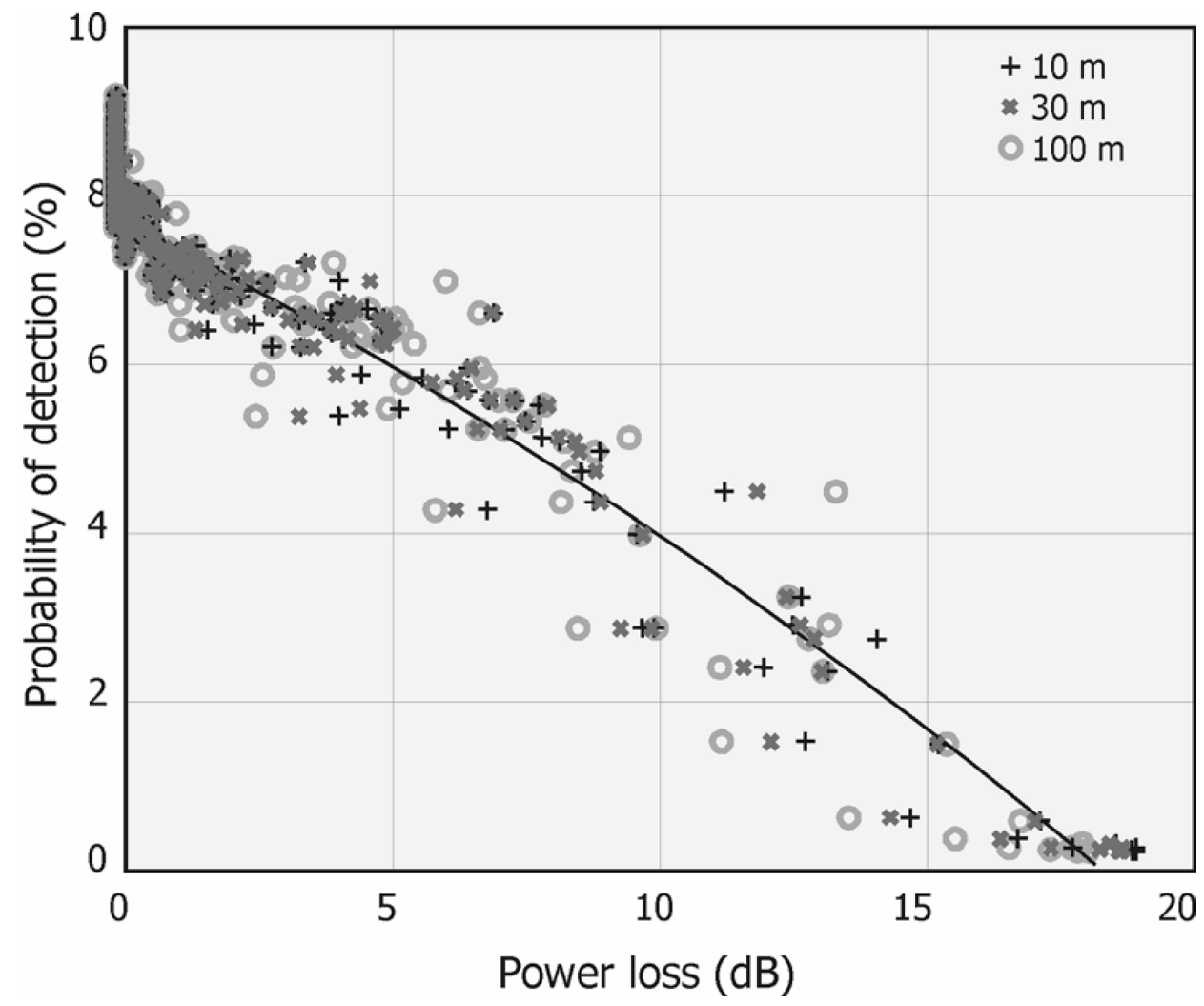

FIG. 7. Scatterplot of (POD) vs power loss for all azimuth angles around Guam. The power loss was calculated using a beam propagation model and DEM data at resolutions of 10, 30, and $100 \mathrm{~m}$. The POD at a range of $50 \mathrm{~km}$ was used in the comparison. The quadratic equation is fit to the 10-m-resolution DEM data.

We wanted to examine the sensitivity of using the $4 / 3$ earth approximation in our beam occultation analysis. This can be done if we assume a spherically stratified atmosphere and the refractive index can be represented by a piecewise linear model of $n$ versus $h$. If $h \ll R$, Eq. (8) can be linearized with respect to $h$ and integrated to determine the radar beam location along the earth's surface as a function of height. The solution to the integral results in the following equation:

$$
\begin{gathered}
s(h)=\left(\frac{\cos \theta_{e}}{1+\beta_{0} R}\right)\left\{\left[R^{2} \sin ^{2} \theta_{e}+2 R\left(1+\beta_{0} R\right) h\right]^{1 / 2}\right. \\
\left.-R \sin \theta_{e}\right\}
\end{gathered}
$$

where $\beta_{0}$ is the gradient of the refractive index, $n$, at the surface and $\theta_{e}$ is the initial elevation angle of the radar (Doviak and Zrnić 1993). Note that $n(0)$ in Eq. (8) is a constant for a given refractive profile and near unity in the atmosphere. Therefore, we have substituted the value of 1 for $n(0)$ in Eq. (10). The ray location at the next height step can be determined by using Eq. (10) and modifying it to reflect the condition at the boundary between the two refractive layers:

$$
\begin{aligned}
& s^{\prime}\left(h^{\prime}\right)=\left(\frac{\cos \theta_{e}^{\prime}}{1+\beta R^{\prime}}\right)\left\{\left[R^{\prime 2} \sin ^{2} \theta_{e}^{\prime}+2 R^{\prime}\left(1+\beta R^{\prime}\right) h^{\prime}\right]^{1 / 2}\right. \\
& \left.-R^{\prime} \sin \theta_{e}^{\prime}\right\},
\end{aligned}
$$

where $s^{\prime}\left(h^{\prime}\right)$ is ground distance from the point of emergence of the ray from the refractive layer below; $\beta$ is the refractive gradient within the layer; $h^{\prime}$ is the height of the ray above the top of the height of the refractive layer below, $h_{b} ; R^{\prime}=R+h_{b}$; and $\theta_{e}^{\prime}$ is the relative elevation angle made between a horizontal plane and the ray emerging at height $h_{b}$. The angle, $\theta_{e}^{\prime}$, is given by

$$
\theta_{e}^{\prime}=\tan ^{-1}(d h / d s),
$$

which can be approximated by the following equation:

$\theta_{e}^{\prime} \cong \tan ^{-1}\left\{\left[R^{2} \sin ^{2} \theta_{e}+2 R h_{b}\left(1+\beta_{b} R\right)\right]^{1 / 2} / R \cos \theta_{e}\right\}$,

where $\theta_{e}$ is the relative elevation angle from the refractive layer below and $\beta_{b}$ is the refractive gradient in the layer below.

The piecewise linear steps can be repeated until the gradient becomes constant or the maximum range from the radar is reached. To examine the assumption of the $4 / 3$ earth ray propagation model, atmospheric soundings 


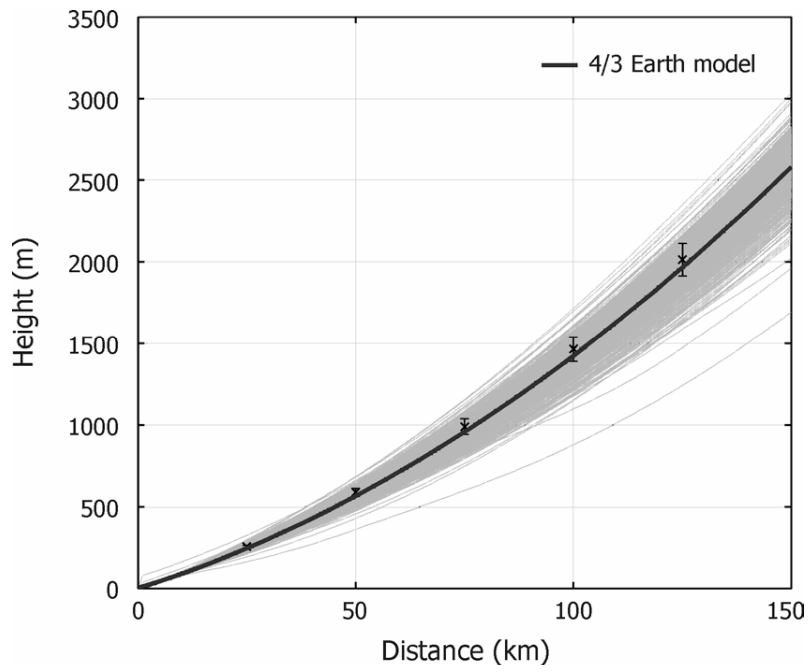

FIG. 8. Beam height results using the index of refraction derived from 2173 Guam soundings (gray). The standard refractive index beam height ( $4 / 3$ earth) calculated for the antenna elevation angle of $+0.5^{\circ}$ is shown as the solid black line. The symbols indicate the mean calculated height and the vertical bars show \pm 1 standard deviation about the mean at ranges of $25,50,75,100$, and $125 \mathrm{~km}$, respectively.

were used to obtain the state of the atmosphere over Guam. For our study, we used over 2000 soundings that were recorded between December 1997 and August 2000 .

The results of using the ray propagations given above for these soundings are given in Fig. 8. The estimated height-distance relationships from the observed refraction profiles are indicated by thin gray lines. The mean profile along with \pm 1 standard deviation is plotted in $25-\mathrm{km}$ intervals. As a reference, the 4/3 earth model is plotted as a black line. The total variation in height from the earth-equivalent model is on the order of $10 \mathrm{~m}$ for distances less than $25 \mathrm{~km}$. At the maximum range of $150 \mathrm{~km}$, the estimated beam heights ranged from 2 to $3 \mathrm{~km}$ (excluding the bottom outlier), indicating that the $4 / 3$ earth model could be off by $500 \mathrm{~m}$ in height at this range.

In most cases, the agreement between the estimated beam height and the $4 / 3$ earth model is very good. At all distances, the mean height lies close to the $4 / 3$ earth line. The plot shows the mean values slightly higher than the standard beam propagation model. The difference ranged from 10 to $49 \mathrm{~m}$ for distances of 25 and $125 \mathrm{~km}$, respectively. The standard deviation about the mean was $8.1 \mathrm{~m}$ at $25 \mathrm{~km}$ and $99 \mathrm{~m}$ at $125 \mathrm{~km}$. The standard refraction beam height estimate was always within 1 standard deviation of the actual sounding estimates and was less than $50 \mathrm{~m}$ from the mean for all distance values.

Except for a few percent of the cases, the 4/3 earth beam height approximation is a good assumption for Guam. Variations in actual beam heights are very sensitive to large gradients of refraction due to temperature inversions and vertical gradients of water vapor in the lowest layer of the atmosphere $(\sim 100 \mathrm{~m})$ (Doviak and Zrnić 1993). Guam has at least two factors minimizing anomalous beam propagation. First, we observed few low-level temperature inversions. The ocean sea surface temperature is warm and relatively constant throughout the diurnal cycle, counteracting any radiational cooling in the boundary layer. Generally, the inversions occurred at around $2-4 \mathrm{~km}$ AGL. At this height, a radar beam is propagating through the varying layers at angles that are insensitive to changes in refraction. Second, the radar is located $\sim 100 \mathrm{~m}$ above the ocean surface so the beam is already above the height where most of the large variations are observed. On the other hand, the atmospheric profiles of temperature and moisture have relatively coarse resolutions using standard rawindsonde data. The instrumentation is not designed to have a fast response time to capture large variations in thin vertical layers. Therefore, our results are probably an optimistic view of the variability of beam propagation with range. High-resolution data from aircraft or lidar observations would be necessary to improve the beam propagation model.

To test the sensitivity of actual height calculations, we implemented the estimated beam heights shown in Fig. 8 into the beam propagation-DEM interaction model. We tested the variation in beam power loss over the AAFB rain gauge site. We chose this azimuth selection because we wanted to determine the variability in beam blockage over the only high-resolution, quality rain gauge installed on Guam, which is relatively unblocked by terrain. The results for this analysis are shown in Fig. 9. This was performed for the $+0.5^{\circ}$ elevation sweep only. Over the AAFB gauge site, the 4/3 earth estimated power loss due to beam blockage was $0.7 \mathrm{~dB}$. We calculated the power loss relative to the standard refraction model. The reason why the number of lines shown in the plot is much smaller than the number of corresponding lines in Fig. 8 is the discretization of the power loss calculations and our assumption that the integration elements $\left(0.1^{\circ} \times 0.1^{\circ}\right)$ are not partially blocked. The plot shows that the power loss increases as the beam propagates over the terrain features represented by the DEM data. It is encouraging to see that most cases have a relative power loss within $5 \%$ of the $4 / 3$ model. The power loss values greater than $5 \%$ correspond to the unusual atmospheric conditions observed in Fig. 8. Based on our results, the 4/3 earth beam height approximation seems valid for most conditions observed on Guam.

\section{Conclusions}

In this paper we demonstrated that GIS-based analysis of radar beam blockage can be a useful tool for climatological and hydrometeorological studies. We developed a simple model for the calculation of the power loss for a radar beam that is due to partial or complete blockage by terrain using DEM data for Guam. To eval- 

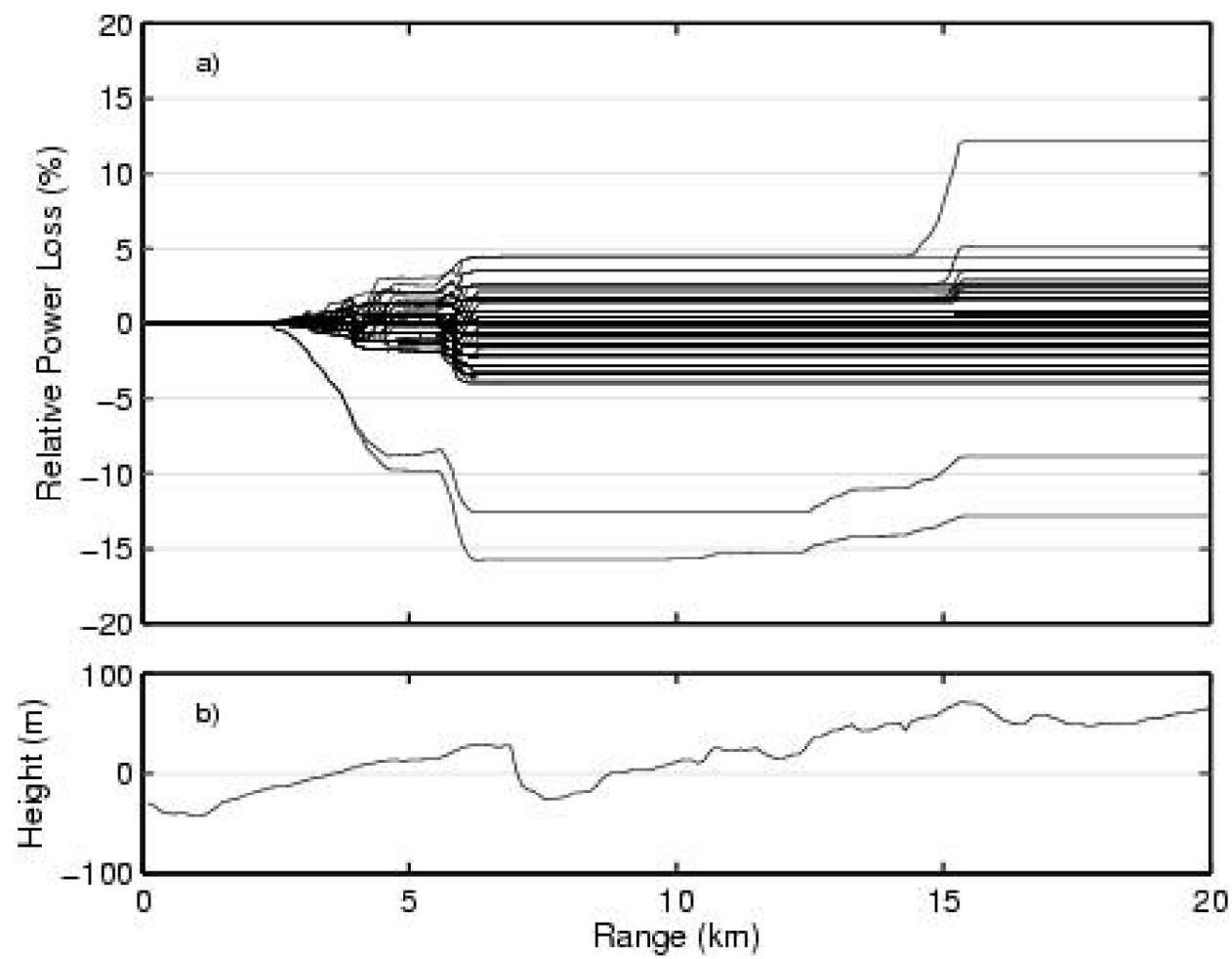

FIG. 9. Relative power loss (a) obtained from the ray propagation model and DEM data over the AAFB rain gauge (azimuth of $43.5^{\circ}$ ) site using the estimated beam heights given in Fig. 8 . The estimated power loss is compared to the power loss using the 4/3 earth approximation. (b) The height of the terrain relative to the height of the PGUA radar antenna. The abrupt changes are caused by the terrain features: Mount Barrigada at about $7 \mathrm{~km}$ from the radar and Mount Santa Rosa some $15 \mathrm{~km}$ away.

uate our model we analyzed actual radar reflectivity data for the same location. Our comparison of the calculated pattern of relative power loss with the POD maps indicates very good qualitative agreement. We also performed a limited quantitative comparison that clearly shows that the two quantities are strongly related.

Our calculations of the power loss and the POD patterns are affected by several sources of uncertainty. We ignored the effects of finite size of beam discretization, beam refraction and multiple reflections, sidelobes, uncertainty in the elevation data, errors in antenna positioning (Bech et al. 2003), errors in the atmospheric sounding data, and the existence of terrain features such as buildings and trees. As our study is not motivated by any particular application that requires detailed analysis, exhaustive uncertainty analysis is beyond the scope of this paper. Still, we succeeded in demonstrating the usefulness of GIS and DEM data analysis for interpretation of radar data that is beyond today's operational practice.

Our results indicate that the resolution of DEM data affects estimates of power loss due to beam blockage. However, this effect is not strong except in very complex terrain. For the Guam WSR-88D at $+0.5^{\circ}$ beam elevation, the power varies from a gain of $+2 \mathrm{~dB}$ to a loss of over $-3.5 \mathrm{~dB}$ for the 100-m DEM resolution compared to the $10-\mathrm{m}$ resolution, depending on the azimuth. The $30-\mathrm{m}$ resolution results show less variation from the $10-\mathrm{m}$ resolution baseline. Lower-resolution data could result in either underestimation or overestimation of the calculated power loss, depending on the location of the sampled elevation data point. Also, including the effects of trees and buildings could further reduce the power in the beam and explain some of the scatter in the empirical results.

The above-mentioned effects strengthen when the beam travels across complex terrain and multiple mountain ranges. The errors due to sampling of the elevation can grow with range from the radar. We could not show this effect on Guam which is too small for such an illustration and does not have multiple mountain ranges. In any case, we think that the lower-resolution data (especially the widely available $30-\mathrm{m}$ data) can be a very useful tool to give estimates of possible blockage for existing sites and for initial surveys for the development of new radar sites. For detailed studies there is a new technology available, that is, airborne laser swath mapping (ALSM), capable of providing data with a resolution on the order of $1 \mathrm{~m}$ (Shrestha et al. 1999). For critical problems and locations we recommend acquiring such data for beam propagation studies.

As this study was motivated by the needs of the 
TRMM project for oceanic rainfall estimation, we used the GIS analysis to define the product map (see Kucera 2002). We identified three main sectors to mask. They correspond to the blocked region to the northeast, the blocked region to the southwest, and the blocked sector in the direction of the apartment building. The blocked sector over Rota in the second sweep is also flagged in the product map. The sector east of the severely blocked Rota sector is partially blocked in the lower sweep. Since for the further ranges we would have to use the lower sweeps in rainfall estimation, we decided to simply block that sector beyond about a range of $70 \mathrm{~km}$. For closer ranges we can use the second sweep, which is not blocked close to the radar. We used this map in all of the analyses of rainfall estimation performance of the Tropical Rainfall Measuring Mission for NASA (see Kucera 2002).

Acknowledgments. This study was partially supported by the National Weather Service under NOAA Grant NA17WH1386 and by NASA under Grants NAG5-4755 and NASA NAG5-9664. We gratefully acknowledge this support. We extend special thanks to Charles "Chip" Guard who assisted in obtaining the Guam DEM data. His organizational skills, knowledge of Guam, and dedication to the common research interests made our trips to Guam lifelong-lasting memories.

\section{REFERENCES}

Andrieu, H., and J. D. Creutin, 1995: Identification of vertical profiles of radar reflectivity for hydrological applications using an inverse method. Part I. Formulation. J. Appl. Meteor., 34, 225239.

Battan, L. J., 1973: Radar Observation of the Atmosphere. The University of Chicago Press, 324 pp.

Bech, J., B. Codina, J. Lorente, and D. Bebbington, 2003: The sensitivity of single polarization weather radar beam blockage correction to variability in the vertical refractivity gradient. $J$. Atmos. Oceanic Technol., 20, 845-855.

Bogush, A. J., 1989: Radar and the Atmosphere. Artech House, 452 pp.

Crum, T. D., R. L. Alberty, and D. W. Burgess, 1993: Recording, archiving, and using WSR-88D data. Bull. Amer. Meteor. Soc., 74, 645-652.

_, R. E. Saffle, and J. W. Wilson, 1998: An update on the NEXRAD program and future WSR-88D support to operations. Wea. Forecasting, 13, 253-262.

Donaldson, R. J., Jr., 1964: A demonstration of antenna beam errors in radar reflectivity patterns. J. Appl. Meteor., 3, 611-623.

Doviak, R. J., and D. S. Zrnić, 1993: Doppler Radar and Weather Observations. Academic Press, $562 \mathrm{pp}$.

Fulton, R. A., J. P. Breidenbach, D.-J. Seo, and D. A. Miller, 1998: The WSR-88D rainfall algorithm. Wea. Forecasting, 13, 377395 .
Hannesen, R., and M. Löffler-Mang, 1998: Improvements of quantitative rain measurements with a C-band Doppler radar through consideration of orographically induced partial beam screening, Proc. COST 75, Locarno, Switzerland, European Commission EUR 18567 EN, 511-519.

Hartree, D. R., J. G. L. Michel, and P. Nicolson, 1946: Practical methods for the solution of the equations of tropospheric refraction. Meteorological Factors in Radio Wave Propagation, Physical Society, 127-168.

Heiss, W. H., D. L. McGrew, and D. Sirmans, 1990: NEXRAD: Next Generation Weather Radar (WSR-88D). Microwave J., 33, 7998.

Krajewski, W. F., G. J. Ciach, and E. Habib, 2003: An analysis of small-scale rainfall variability in different climatological regimes. Hydrol. Sci. J., 48, 151-162.

Kruger, A., and W. F. Krajewski, 1997: Efficient storage of weather radar data. Software Practice Experience, 27, 623-635.

Kucera, P. A., 2002: Performance characteristics of a spaceborne precipitation radar over an ocean validation site. Ph.D. dissertation, The University of Iowa, Iowa City, IA, $338 \mathrm{pp}$.

Kummerow, C., and Coauthors, 2000: The status of the Tropical Rainfall Measuring Mission (TRMM) after two years in orbit. J. Appl. Meteor., 39, 1965-1982.

Meneghini, R., and T. Kozu, 1990: Spaceborne Weather Radar. Artech House, 199 pp.

Nelson, B., W. F. Krajewski, A. Kruger, J. A. Smith, and M. L. Baeck, 2003: Archival precipitation data set for the Mississippi River basin: Development of a GIS-based data browser. Comput. Geosci., 29, 595-604.

Pellarin, T., G. Delrieu, G. M. Saulnier, H. Andrieu, and J. D. Creutin, 2002: Hydrologic visibility of weather radars operating in mountainous regions: Case study for the Ardèche catchment (France). J. Hydrometeor., 3, 539-555.

Press, W. H., S. A. Teukolsky, W. T. Vetterling, and B. P. Flannery, 1992: Numerical Recipes in C: The Art of Scientific Computing. 2d ed. Cambridge University Press, 994 pp.

Probert-Jones, J. R., 1962: The radar equation in meteorology. Quart. J. Roy. Meteor. Soc., 88, 485-495.

Rinehart, R. E., 1991: Radar for Meteorologists. R. E. Rinehart, 334 $\mathrm{pp}$

Shrestha, R. L., W. E. Carter, M. Lee, P. Finer, and M. Sartori, 1999: Airborne laser swath mapping: Accuracy assessment for surveying and mapping applications. J. Amer. Congr. Surveying Mapping, 59, 83-94.

Simpson, J., R. F. Adler, and G. North, 1988: A proposed tropical rainfall measuring mission (TRMM). Bull. Amer. Meteor. Soc., 69, 278-295.

— W. W.-K. Tao, C. Kummerow, and R. Adler, 1996: On the Tropical Rainfall Measuring Mission (TRMM). Meteor. Atmos. Phys., 60, 19-36.

USGS, 2000: U.S. GeoData digital elevation models. USGS Fact Sheet 040-00, EROS Data Center, Sioux Falls, SD, 2 pp.

Ward, P. E., S. H. Hoffard, and D. A. Davis, 1965: Hydrology of Guam. Geological Survey Prof. Paper 403-H, U.S. Government Printing Office, Washington, DC, $28 \mathrm{pp}$.

Westrick, K. J., C. F. Mass, and B. A. Colle, 1999: The limitations of the WSR-88D radar network for quantitative precipitation measurement over the coastal western United States. Bull. Amer. Meteor. Soc., 80, 2289-2298.

Young, C. B., B. R. Nelson, A. A. Bradley, J. A. Smith, C. D. PetersLidard, A. Kruger, and M. L. Baeck, 1999: An evaluation of NEXRAD precipitation estimates in complex terrain. J. Geophys. Res., 104, 19 691-19 703. 